\title{
Enzimas cerebrales y psicofarmacología del alcohol
}

\author{
Escarabajal, M. D.
}

Universidad de Jaén

\section{RESUMEN}

La existencia de un metabolismo cerebral del etanol es desde hace más de una década motivo de controversia, por lo que el trabajo que se presenta tiene, entre otros objetivos, realizar un balance y revisión actualizados de los aspectos más relevantes relacionados con la presencia de acetaldehído, el primer metabolito del etanol, en el cerebro. Para ello se presenta la evidencia bioquímica, conductual y genética existente sobre la presencia, localización, y función de los sistemas enzimáticos implicados en la biotransformación de etanol a acetaldehído y de la posterior transformación de este último a acetato.

Los resultados expuestos conducen de forma concluyente a dos aspectos fundamentales, por una parte, la viabilidad de un metabolismo cerebral del alcohol, dado que los sistemas formados por la catalasa y la ALDH cerebrales son la maquinaria enzimática necesaria y suficiente para el oxidación de alcohol a acetaldehído, y la posterior degradación de este a acetato y, por otra, que estos sistemas enzimáticos mediarían algunas de las conductas inducidas por etanol mediante variaciones en la concentración de acetaldehído en el cerebro, lo que supone la implicación del acetaldehído en algunas de las acciones psicofarmacológicas atribuidas tradicionalmente al etanol.

Palabras clave: alcohol, metabolismo, aldehído deshidrogenasa, alcohol deshidrogenasa, catalasa, acetaldehído, revisión.

\section{ABSTRACT}

The possibility of a brain metabolism of ethanol has been a controversial issue for over a decade. This paper reviews and updates the most relevant questions on the occurrence of acetaldehyde, the primary metabolite of ethanol, in brain. We present here the biochemical, behavioural and genetic results about the existence, location and role of the enzymatic systems involved in the metabolism of ethanol into acetaldehyde and its subsequent conversion to acetate.

The results show two conclusions: first, a brain metabolism of alcohol may exist, because brain catalase and ALDH are the necessary and sufficient enzymatic systems to oxidize ethanol into acetaldehyde and, in turn, acetaldehyde into acetate; second, these enzymes intervene in some ethanol-induced behaviours due to their ability to regulate levels of acetaldehyde in brain. Acetaldehyde can thus be assumed to be responsible for some of psychopharmacology behaviours traditionally attributed to ethanol.

Key words: alcohol, metabolism, aldehyde dehydrogenase, alcohol dehydrogenase, catalase, acetaldehyde, review.

\section{1. ¿EXISTE UN METABOLISMO CEREBRAL DEL ETANOL?}

$\mathbf{P}$ lantear que el alcohol disfruta de un metabolismo periférico en el que interviene de forma fundamental el hígado es un hecho por todos conocido, sin embargo, y dado que varios estudios apuntan hacia la idea de un metabolismo también cerebral, tema no exento de polémica y controversia, en esta revisión se tratará de exponer las pruebas existentes que avalan este metabolismo central del etanol. En este sentido, la existencia de un metabolismo del etanol en el cerebro tiene como requisitos básicos que esta estructura contenga los enzimas necesarios tanto para su oxidación como para la posterior degradación de sus metabolitos (acetaldehído y acetato), con ese objetivo se presentan los sistemas enzimáticos cerebrales existentes y su viabilidad como maquinaria enzimática necesaria y suficiente para desarrollar esa transformación a nivel cerebral. 
Por otra parte, se intentará determinar a partir de los datos experimentales sobre este tema, si es el acetaldehído el responsable directo de los efectos psicofarmacológicos que se observan tras la ingestión de etanol o si por el contrario es el alcohol el primer y último mediador de sus acciones.

En relación con este último punto, cabe señalar que la mediación del acetaldehído en algunos de los efectos reforzantes del etanol es actualmente motivo de controversia debido a una serie de problemas metodológicos y fisiológicos que dificultan su aceptación. Entre ellos está, que la detección de acetaldehído en el Sistema Nervioso Central (SNC) tras el consumo de cantidades normales de etanol es prácticamente nula $(1,2,3)$ debido a que la acción enzimática Ilevada a cabo por la aldehído deshidrogenasa (ALDH) hepática no permite que prácticamente ningún acetaldehído salga del hígado y alcance el cerebro. Además, para aquellas cantidades de acetaldehído que pudiesen escapar del hígado existe una barrera enzimática cerebral formada por la $\operatorname{ALDH}(4,5)$ que ejerce su protección sobre el cerebro, al menos para concentraciones moderadas de alcohol. De este modo, si el acetaldehído no puede atravesar la protección de la ALDH en el cerebro ¿cómo podría el acetaldehído ser el responsable de los acciones psicofarmacológicas del etanol? La respuesta que dan algunos autores $(6,7)$ es que es necesario que el acetaldehído se forme directamente en el cerebro, hecho que permitiría determinar si está o no implicado en las propiedades psicofarmacológicas del etanol.

En el punto siguiente se presentan las pruebas existentes a favor de la localización cerebral del acetaldehído a partir del metabolismo del etanol en el SNC.

\section{SISTEMAS ENZIMÁTICOS.}

La curva metabólica del etanol requiere enzimas tanto para su oxidación en acetaldehído (esta conversión está mediada por tres sistemas enzimáticos la alcohol deshidrogenasa (ADH), el citocromo P-450 y la catalasa); como para la degradación del acetaldehído en acetato (proceso llevado a cabo por la ALDH).

El metabolismo periférico ya sea el hepático o el que se produce en otros órganos (estómago, riñón, etc.) se conoce y no reviste ningún problema (8, para una revisión reciente). Sin embargo, plantear la existencia de un metabolismo central y un papel para el acetaldehído formado a nivel cerebral no ha tenido la misma suerte.

En los apartados siguientes se exponen datos sobre los enzimas implicados en el metabolismo del etanol, que sugieren que el acetaldehído puede ser formado directamente en el tejido cerebral y que estos enzimas cerebrales poseen una función reguladora sobre las conductas inducidas por etanol.

\subsection{ALCOHOL DESHIDROGENASA (ADH)}

\subsubsection{Localización cerebral.}

Como se ha comentado anteriormente la conversión de etanol a acetaldehído la catalizan tres sistemas enzimáticos como son la $\mathrm{ADH}$, el citocromo P-450, más concretamente la forma 2E1 y, la catalasa.

La presencia de la actividad específica de ADH en el cerebro fue demostrada por primera vez por Raskin y Sokoloff (9). Sin embargo, datos posteriores indicaron que en el cerebro la actividad de este enzima era muy pobre $(10,11)$. Posteriormente, experimentos con inmunohistoquímica permitieron la detección de $\mathrm{ADH}$ en el citoplasma de algunas neuronas de la corteza cerebral, sobre todo en los cuerpos mamilares, la sustancia gris periacueductal, el hipotálamo, el tallo infundibular de la pituitaria y en células cerebelares de Purkinje $(12,13)$.

En 1975, Tabakoff y Von Wartburg (14) aislaron en ratas una $\mathrm{ADH}$ cerebral que presentaba algunas características similares a la existente en el hígado. El enzima fue detectado en el citosol y el núcleo, pero no se encontró ni en los microsomas ni en las mitocondrias, lo que indica que esta localización se limita al citoplasma neuronal y solo en algunas neuronas. Sin embargo, esta restricción en la localización de ADH a un pequeño número de neuronas en el SNC explicaría la dificultad para demostrar la existencia del enzima en la totalidad de los homogenados cerebrales. Además, también podría indicar la importancia de la actividad enzimática a nivel local aunque globalmente su actividad sea baja. Por otra parte, la significación de la localización de $\mathrm{ADH}$ depende de los isozimas que estén presentes. En relación con esto, existen diversos isozimas de $\mathrm{ADH}$ que se diferencian por la afinidad que tengan al etanol y por la facilidad con la que el isozima puede ser inhibido por pirazol (15).

En el hígado es principalmente el isozima ADH de la clase I el que interviene en la oxidación del etanol, sin embargo, en el cerebro su acción es muy limitada $(11,16)$. Además, en el SNC de humanos y roedores la única forma presente de ADH en cantidades significativas es el isozima de la clase III $(12,17,18,19)$.

La distribución de la ADH III en el cerebro es amplia aunque su mayor concentración se localiza en la capa subependimal y las áreas perivasculares de la corteza cerebral, el subcórtex, el tallo cerebral y el cerebelo (20). Entre sus características presenta una muy baja afinidad por el etanol ( $\mathrm{Km}$ mayor de 2,5 M) y, como consecuencia de esto, sus oportunidades para 
oxidar a esta sustancia están bastante limitadas. De hecho, su papel en la oxidación cerebral de etanol no es importante ni siquiera bajo una intoxicación severa con alcohol $(19,20)$. En este sentido, aunque se detectó actividad de ADH en capilares sanguíneos, astrocitos y neuronas de varias regiones cerebrales, cuando se utilizó etanol como sustrato no se detectó ninguna actividad en el cerebro, mediante histoquímica, para la ADH.

Encontramos así que de los tipos de ADH existentes y relacionadas con el etanol, la ADH I parece estar ausente del cerebro mientras que la ADH III, aunque está presente, no desempeña una función determinante en el metabolismo cerebral del etanol, debido a su baja afinidad por el etanol incluso aunque existan niveles elevados de esta sustancia en plasma (21), sirva como ejemplo que la actividad de la ADH cerebral bovina fue la cuatromilésima parte de la actividad de ese mismo enzima en el hígado (22). Estos datos abren la posibilidad de que sean otros enzimas los encargados de metabolizar el etanol en el cerebro.

Sin embargo, antes de finalizar este punto cabría señalar que una posible función de la ADH cerebral sea la oxidación de alcoholes grasos de larga cadena (20). También ha mostrado su función en la conversión de retinol a retinaldehído el cual es convertido a ácido retinóico, por la ALDH, que está implicado en el desarrollo del tubo neural en los embriones de vertebrados (23), por lo que los defectos del tubo neural inducidos por el etanol, pueden ser debidos a la oxidación del retinol por la inhibición del etanol $(23,24)$. Por otra parte, la clase III de la ADH es idéntica a una deshidrogenasa de formaldehído dependiente de glutationa (25), existiendo una ADH con propiedades similares en el cerebro de ratón (19), cuya presencia podría reflejar la necesidad de una sustancia que actúe como neutralizador del formaldehído para la citoprotección (26). Además, no se excluye la posibilidad de que la $\mathrm{ADH}$ oxide etanol en algunas células específicas del cerebro. Así, la ADH cerebral puede actuar reduciendo el acetaldehído, producido por el citocromo P-450 o por la catalasa in situ, a etanol y también eliminar el excedente de acetaldehído en las células que poseen poca actividad de ALDH (22).

\subsection{CITOCROMO P-450 2E1.}

\subsubsection{Localización cerebral.}

La presencia de citocromos pertenecientes al sistema microsomal de oxidación del etanol (MEOS), concretamente del CYP-450, en el cerebro fue observada por primera vez en 1977 (27). Más tarde, la habilidad para metabolizar xenobióticos se demostró para cerebros de ratón (28), de rata (29) y de sujetos huma- nos $(30,31)$; y se detectaron $n$ rata varias formas de CYP-450, aunque sus cantidades por gramo de tejido eran muy pequeñas $(29,32)$. Sin embargo, los estudios inmunohistoquímicos ponen de manifiesto la presencia de un amplio número de formas del CYP-450 en varias poblaciones de glía y neuronas en diversas regiones del cerebro, mostrando que células individuales pueden contener cantidades significativas de estos enzimas $(33,34)$.

La forma específica del citocromo P-450 relacionada más directamente con el metabolismo del etanol es el citocromo P-450 2E1 (CYP-450 2E1). Este y otros subtipos, localizados en el cerebro por Morgan et al., (35), se encuentran en células gliales, neuronas y vasos sanguíneos de todas las regiones cerebrales $(32,36)$, encontrándose la máxima inmunoreactividad en las neuronas piramidales del córtex frontal y el hipocampo, en neuronas del estriado, sustancia negra, núcleo pontino, núcleo olivar superior, núcleo de los nervios trigémino y facial, sustancia gris central y en la formación reticular.

EI CYP 2E1 es inducido en el hígado por etanol y esa misma inducción ha sido puesta de manifiesto en el cerebro tanto tras un tratamiento crónico $(37,38$, $39,40)$ como tras administraciones agudas de etanol $(32,41)$. Esta inducción es de gran importancia porque indirectamente podría indicar un metabolismo cerebral del etanol ya que se sabe que el CYP 2E1 hepático normalmente es inducido por los substratos a los que metaboliza.

Sin embargo, aunque no hay duda de la presencia e inducción del CYP 2E1 en estructuras cerebrales tras el tratamiento con etanol $(16,42)$, ni de la contribución potencial para la neurotoxicidad del etanol a través de la producción de radicales libres; los estudios existentes adolecen del curso temporal de la inducción, y se hace necesaria la propuesta de una ruta de síntesis que produjera concentraciones significativas de acetaldehído en el cerebro (43). Estos aspectos dificultan que este sistema enzimático sea el candidato a mediar en el metabolismo central del alcohol.

\subsection{CATALASA ENCEFÁLICA.}

\subsubsection{Localización cerebral.}

La catalasa es un enzima cuya función principal es eliminar la intoxicación provocada por el peróxido de hidrógeno $\left(\mathrm{H}_{2} \mathrm{O}_{2}\right)$ (44), su presencia en el cerebro ha sido estudiada bioquímica, histoquímica e inmunohistoquímicamente en adultos $(45,46,47,48)$, en cerebros en desarrollo (49) y en cultivos de células cerebrales de ratas fetales $(50,51,52,53)$. Su actividad se localiza en los microperoxisomas $(45,54)$ prin- 
cipalmente en los pericariones de las neuronas aminérgicas (48), siendo las regiones cerebrales de máxima actividad catalítica el núcleo del tracto solitario, cerca del área postrema donde se sitúan las neuronas adrenérgicas y noradrenérgicas, el núcleo del rafe dorsal y el núcleo arqueado del hipotálamo. En estas regiones más de la mitad de las neuronas son catalasa positivas y en algunas de ellas la densidad de este enzima es mayor que en los hepatocitos. Existe también actividad de la catalasa en la sustancia blanca de la glía en todas las regiones cerebrales, detectando una mayor concentración en los ependimocitos que recubren el tercer ventrículo, especialmente en la zona cercana a la eminencia media $(48,55)$. Esta distribución enzimática en la que las células positivas para la catalasa se encuentran en una pequeña parte del conjunto cerebral podría explicar la baja actividad para el enzima que se ha encontrado en homogenados cerebrales $(46,56,57)$.

En relación con la degradación de acetaldehído los resultados han puesto de manifiesto que la localización de neuronas que contienen catalasa contrasta con aquellas que contienen $\operatorname{ALDH}(48,58)$ ya que este último enzima presenta una amplia distribución en el cerebro, y desarrolla, al mismo tiempo, una baja actividad en los pericariones de las neuronas aminérgicas, especialmente las catecolaminérgicas tanto en cerebro de rata (5) como en humanos (59). Sin embargo, y como no se conocen los niveles cerebrales mínimos de acetaldehído necesarios para ejercer sus efectos, aunque la cantidad total de acetaldehído formado es pequeña, existe la posibilidad de que se den concentraciones suficientes para provocar modificaciones en determinados centros cerebrales implicados en el reforzamiento.

En este sentido, por parte de algunos autores (48, $60,61,62)$ se ha planteado que el sistema catecolaminérgico cerebral podría estar implicado en la mediación de los efectos del acetaldehído en el cerebro. Así, las neuronas aminérgicas podrían presentar una insuficiencia en la eliminación de acetaldehído lo que provocaría una acumulación local de acetaldehído tras la administración de alcohol. De este modo, la localización de la catalasa en neuronas aminérgicas, pobres en ALDH, provocaría una oxidación selectiva del etanol y una acumulación de acetaldehído en neuronas aminérgicas, activación de estas neuronas y consecuente estimulación de, por ejemplo, el consumo de alcohol. Esto podría explicar el papel de la catalasa cerebral y del acetaldehído originando acciones psicofarmacológicas centrales del etanol y también indicaría el posible mecanismo que implica al sistema de neuronas aminérgicas en los efectos centrales del alcohol.

En resumen, los datos presentados hasta este momento podrían indicar que aunque al igual que ADH y MEOS, el enzima catalasa presenta una serie de dificultades en la mediación del metabolismo cen- tral del etanol, es también el más firme candidato en ese metabolismo cerebral. Sin embargo, a pesar de la cantidad de trabajos en los que se confirma a nivel cerebral la presencia de los sistemas que oxidan etanol, la pregunta en torno a la oportunidad para llevar a cabo una oxidación significativa del etanol en el SNC sigue abierta y continúa siendo tema de debate $(7,63)$, por ello, a continuación se expone un posible mecanismo de la mediación de catalasa en la oxidación del etanol a nivel cerebral.

\subsubsection{Catalasa y metabolismo del etanol.}

Este enzima desempeña una doble función peroxidática y catalítica, dependiente de la concentración de peróxido existente en el organismo (64), dándose, por ejemplo, la oxidación de algunos alcoholes a baja concentración de peróxido. En relación con esto, el mecanismo de reacción entre etanol y catalasa podría ser el siguiente:

El enzima catalasa reacciona con el peróxido de hidrógeno formándose una molécula de catalasa activada que se ha denominado compuesto 1. Este compuesto 1 podría a su vez reaccionar con etanol y obtener como productos de la reacción acetaldehído y agua.

Compuesto 1 (catalasa $+\mathrm{H}_{2} \mathrm{O}_{2}$ ) + etanol $\rightarrow$ acetaldehído $+\mathrm{H}_{2} \mathrm{O}$

Se han desarrollado varios trabajos in vivo e in vitro que apoyan la posibilidad de que la reacción entre compuesto 1 y etanol se produzca en el cerebro. Así, se ha obtenido que el pretratamiento con etanol previene al enzima de la acción de sus inhibidores, indicando una competición entre el etanol y dichos inhibidores por el compuesto 1. Por otra parte, también se ha confirmado la generación y presencia de peróxido en el cerebro (65), de este modo, catalasa y peróxido de hidrógeno se unirían para formar el compuesto 1 , y cuando este compuesto 1 reacciona con algún inhibidor, su acción genera una destrucción irreversible de la actividad de la catalasa cerebral, que es dependiente de las dosis de etanol e inhibidor utilizadas. Esta protección que ejerce el etanol sobre la inhibición de la actividad de la catalasa nos proporciona una prueba indirecta de la competición que puede darse entre etanol e inhibidor por el lugar activo del compuesto 1.

Por otra parte, Cohen et al., (66) presentaron la primera evidencia para la oxidación cerebral de etanol a acetaldehído por la catalasa cerebral. Además, Tampier y Mardones (67) con homogenados cerebrales de rata, obtuvieron que la adición de 3-amino-1,2,4-triazol al cultivo reducía la oxidación de etanol en un $50 \%$. Estudios posteriores $(56,57,65)$ han confirmado estos resultados indicando la formación del acetaldehído en cerebros de ratas sin encontrar participación de $A D H$ 
o CYP-450, obteniendo incrementos en la concentración de acetaldehído si se añadía al cultivo peróxido de hidrógeno o un sistema generador del mismo.

Así, aunque los niveles endógenos de peróxido de hidrógeno son el factor limitante en la oxidación cerebral de etanol por la catalasa $(56,57,65)$, existen numerosos candidatos a actuar como fuente de peróxido de hidrógeno. Por ejemplo, la superóxido, cuando actúa sobre la superóxido dismutasa, produce peróxido de hidrógeno (72), la monoaminooxidasa que lo genera en el proceso de deaminación oxidativa de las aminas biogénicas o, el ácido ascórbico (73).

Los estudios con cultivos de neuronas hipotalámicas tratadas con etanol, propanol y butanol, indican que la estimulación de la liberación de ^-endorfinas solo la produjo el etanol, y al pretratar a estas neuronas con inhibidores de la catalasa se inhibía la liberación y la producción del acetaldehído de forma dependiente de la dosis de inhibidor utilizada (68), lo que sugiere que el acetaldehído mediado por la catalasa fue responsable del aumento en la liberación de *-endorfinas por la estimulación de etanol.

Existen otras pruebas en el mismo sentido (69), de modo que se produce una acumulación de acetaldehído, en diferentes estructuras cerebrales tras ser incubadas con etanol, que es eliminada parcialmente por el pretratamiento con inhibidores. Además, el tratamiento crónico con inhibidores de la catalasa supone una disminución dosis dependiente, en la catalasa encefálica (70).

Cabría señalar que las concentraciones de etanol utilizadas en los trabajos presentados son las mismas que se pueden alcanzar en el cerebro de rata tras la ingesta de etanol y son suficientes para producir efectos farmacológicos (71). Estos y otros resultados relacionados apoyarían el papel de la catalasa en la regulación del metabolismo del acetaldehído en el cerebro.

Pese a esta evidencia sobre la formación cerebral de acetaldehído, todavía habría que determinar si este acetaldehído cerebral puede tener o no consecuencias conductuales. Para intentar determinarlo a continuación se presentan varios trabajos que relacionan la catalasa con diferentes efectos producidos por el etanol.

Datos relacionados con el consumo de alcohol muestran correlaciones positivas entre la actividad total de la catalasa cerebral y el consumo voluntario de etanol en roedores $(74,75,76)$. Además, si la actividad de la catalasa tiene un papel en los efectos conductuales del etanol, la afinidad diferencial al etanol de muchas cepas de ratas y ratones podría estar mediada por la actividad de la catalasa, aspecto que también se observa ya los animales con mayor preferencia y consuno de etanol tienen menores niveles de catalasa y menores niveles de acetaldehído en sangre $(77,78)$.

Otra estrategia ha sido la manipulación de la catalasa mediante inhibidores de su actividad, obteniendo que en animales pretratados con 3-amino-1,2,4-triazole, disminuyó la ingesta y la preferencia por el etanol (79), el tiempo de narcosis y la mortalidad inducida por etanol $(80,81,82)$, la depresión motora (83), y se bloqueó el condicionamiento de aversión al sabor (CAS) inducido por etanol pero no por otras drogas como morfina y cloruro de litio (84). Resultados similares se han obtenido en ratones con este y otros inhibidores $(76,85,86,87)$.

Estos resultados, sugieren que el tratamiento con inhibidores de la catalasa produce modificaciones sobre algunos efectos conductuales del etanol, efectos que podrían estar causados por una reducción en la formación del acetaldehído central.

Para determinar la mediación de la catalasa en el metabolismo cerebral del etanol en estudios con poblaciones humanas. Estos trabajos (89) han replicado muchos de los resultados obtenidos con animales (75), mostrando correlaciones positivas entre actividad de la catalasa cerebral y la eritrocitaria antes, durante y después de la exposición al etanol, siendo la catalasa era la variable predictora con mayor significación de las evaluadas (88). Además, en estos experimentos se encuentra un fuerte apoyo a la idea de que la actividad de la catalasa en humanos y especialmente en los que tienen una historia familiar de alcoholismo, puede representar un marcador biológico de la propensión de los individuos, llamados de alto riesgo, a consumir etanol.

Así, los resultados derivados de la población humana apoyan de la idea de que la actividad de la catalasa puede tener una significación positiva en la determinación del consumo de alcohol en humanos, y afianza la propuesta de la catalasa como un marcador viable para la propensión a consumir etanol en las personas.

El siguiente punto contiene los resultados obtenidos que dan apoyo a la idea de que el acetaldehído se puede formar directamente en el cerebro mediante la acción de sistemas enzimáticos presentes en esta estructura.

\subsubsection{La presencia de acetaldehído en el cerebro.}

\subsubsection{Generalidades.}

Aunque el acetaldehído se ha considerado el responsable de los efectos aversivos que produce el etanol (90, para una reciente revisión), existe otro cuerpo de estudios que apoya la mediación del acetaldehído central en las acciones psicofarmacológicas del etanol. Sin embargo, la detección de acetaldehído cerebral ha sido difícil (2) ya que este metabolito parece ser detectable solo cuando se da una intoxicación importante de etanol o tras la administración de inhibidores del metabolismo del acetaldehído (91). Pero esta escasez de resultados concluyentes sobre la presencia de acetaldehído en el cerebro, no elimina su 
existencia, hecho que puede ser particularmente relevante dado que las cantidades necesarias de esta sustancia para producir efectos farmacológicos siguen sin conocerse.

En este sentido, se ha intentado demostrar la habilidad de los cultivos de astrocitos para producir acetaldehído a partir del etanol (92), exponiendo los cultivos a diversas concentraciones de etanol. Los resultados indicaron que se produce un metabolismo significativo del etanol en los cultivos y, aunque la producción es menor que la observada en los hepatocitos, se corresponden con niveles biológicamente activos de acetaldehído. Estudios más recientes (53) aportan datos que confirman que el acetaldehído está presente después de la ingesta de etanol tanto en cerebros adultos (91) como en cerebros fetales (93).

En este sentido, la utilización de homogenados de cerebro de ratas inmaduras ha puesto también de manifiesto la posibilidad de una generación de acetaldehído vía una reacción mediada por la catalasa, planteando además que este metabolito del etanol puede ser producido in vivo en ese sistema (53). Así, cuando los homogenados fueron incubados con inhibidores de la catalasa -como el 3-amino-1,2,4-triazole o la azida sódica-, se obtuvo un bloqueo en la formación de acetaldehído, mientras que inhibidores de la ADH o del P-450, no disminuyeron la producción de este metabolito, a partir de lo cual, se podría afirmar que ni la vía oxidativa del CYP 2E1 ni la de la ADH son fuentes de acetaldehído medible en el cerebro. Además, estos resultados van en la misma línea que aquellos (57) que informaron de que los homogenados de cerebros neonatales generan acetaldehído, y este es producido probablemente en el cerebro de ratas fetales y neonatales a partir de la oxidación del etanol mediada por la catalasa. Otros estudios han implicado la acción del acetaldehído en la secreción de $\boldsymbol{*}$-endorfinas en neuronas hipotalámicas $(68,94)$, pudiendo afirmar a partir de los resultados obtenidos que el acetaldehído mediaría parte del efecto estimulatorio del etanol sobre la secreción de -endorfinas.

Como se ha visto, aunque existe un eficiente sistema de oxidación de acetaldehído en la BHE que mantiene la entrada de este acetaldehído periférico dentro del cerebro en un nivel mínimo (95); existen también diversas evidencias que indicarían que el tejido cerebral metaboliza etanol a acetaldehído.

\subsubsection{Pruebas genéticas.}

La existencia de acetaldehído cerebral se ha determinado también mediante diferentes cepas de ratas y ratones tanto desde estudios in vitro como mediante diferencias conductuales. Así, ratas UChB muestran una capacidad de oxidación de acetaldehído menor que la de las ratas UChA (96), obteniéndose la mayor cantidad de acetaldehído en el córtex diencefálico de estas últimas (97), al mismo tiempo, homogenados cerebrales de estas cepas mostraron que las UChB tienen mayor capacidad de oxidación por una afinidad mayor en su ALDH por el nicotinamida-adenín-dinucleótido (NAD) (98).

Otros estudios con cepas de alto y bajo consumo voluntario de alcohol (Alko alcohol-AA- y Alko nonalcohol -ANA- respectivamente) oxidan diferencialmente el etanol. Así, en ratas $A A$ se observó un rango mayor para la oxidación que en las ANA, demostrando también que ratas $A$ A presentaban niveles menores de acetaldehído que las ANA durante la oxidación de etanol (99).

Por su parte, los datos con sujetos humanos indican tras la ingesta de etanol el acetaldehído se encuentra en concentraciones mayores en alcohólicos y bebedores severos comparados con bebedores sociales o abstemios (100).

Sin embargo, no deberíamos obviar que, como señala Hunt (43), aunque el papel del acetaldehído en las acciones del etanol es prometedor su apoyo es indirecto y circunstancial implicando estudios correlacionales y evidencia farmacológica.

Hasta este momento los datos presentados ponen de manifiesto que cuando tras la administración de etanol el acetaldehído se encuentra en la periferia a altas concentraciones, puede inducir reacciones aversivas tanto en humanos como en animales (101). Por otra parte, se ha mostrado también que este metabolito está relacionado con conductas como, por ejemplo, la auto administración en el cerebro (intracerebro ventricular -ICV- o en el área tegmental ventral) de esa misma sustancia $(102,103,104)$, además, produce preferencia de lugar administrado tanto ICV como intraperitonealmente $(105,106)$, hechos que lo relacionan con propiedades reforzantes positivas. Sin embargo, aunque parece que el acetaldehído puede actuar como un agente positivo o aversivo por sí mismo, no existe acuerdo en su función mediadora de algunos de los efectos del etanol.

Como se ha visto en los apartados anteriores, esto ha llevado a realizar numerosas investigaciones orientadas a demostrar la presencia de acetaldehído en el cerebro tras la exposición al etanol.

Por último, un papel significativo del acetaldehído en las acciones del etanol en el cerebro requiere la presencia de este metabolito en el cerebro después del consumo de etanol en cantidades consistentes con las que se utilizan en los experimentos que muestran cambios biológicos o conductuales, pero por el momento, los datos son poco claros. Lo que lleva a plantear la necesidad de una vía en la que el acetaldehído pueda ser cuantificado en el cerebro in vivo después de la administración de etanol.

Por otra parte, los datos presentados hasta este momento sobre el metabolismo central del etanol 
requieren el tratamiento de los enzimas cerebrales implicados no solo en la producción central de acetaldehído sino también de los enzimas responsables de la eliminación de esta sustancia. Por ello en el siguiente apartado se presentan los datos conocidos sobre la ALDH cerebral, su localización, funciones y su posible implicación en la mediación de algunas de las acciones relacionadas con el etanol y más directamente con el acetaldehído.

\subsection{ALDEHÍDO DESHIDROGENASA (ALDH).}

\subsubsection{Localización cerebral.}

La presencia de ALDH en el cerebro ha sido demostrada para diferentes especies de mamíferos como el ser humano (107) y la rata (108). Y los estudios realizados para determinar su localización cerebral han puesto de manifiesto que está ampliamente distribuida por todo el cerebro $(48,109)$; en este sentido, las neuronas de ALDH se distribuyen en el cerebro como sigue, un $40 \%$ se localizan en el núcleo arqueado hipotalámico y un $88 \%$ en las células cerebelares de Purkinje (109). Una distribución más exacta muestra una actividad moderada de este enzima en los capilares y alrededor de los astrocitos y una actividad elevada en los ependimocitos que recubren las cavidades cerebrales y los del plexo vascular $(5,109)$.

El estudio de la localización y actividad de ALDH en diferentes cepas de ratas y ratones indica mayor actividad en neuronas del tracto mesencefálico del núcleo del nervio trigémino y en las motoneuronas de la médula espinal. Mientras que se detectó una menor actividad en el córtex somatosensorial (58, 113), encontrando diferencias en las células cerebelares de Purkinje entre las líneas de ratas y ratones sensibles y resistentes al alcohol, mostrando estos últimos, niveles estadísticamente mayores de ALDH.

Por su parte, la fracción mitocondrial es en la que se da la mayor actividad enzimática, encontrándose neuronas de ALDH mitocondrial en todas las regiones cerebrales desde las estructuras donde es más abundante -oliva inferior-, hasta las que la tienen en menor cantidad, como el estrato piramidal del hipocampo y la sustancia negra (109)

Además, el estudio de regiones cerebrales en ratas y humanos, en medios tratados con aldehídos, pone de manifiesto que la actividad la fracción mitocondrial de ALDH fue alrededor del 50\% de la actividad total, frente a un 10-15\% para la fracción citosólica y un $10 \%$ para la microsomal $(107,110)$. Esta misma tendencia se ha obtenido en estudios conductuales en los que se obtuvieron altas correlaciones positivas entre ingesta voluntaria de etanol en ratas y ALDH cerebral mitocondrial (111).
Por otra parte, la distribución subcelular del enzima en el cerebro humano es similar a la del hígado humano, que también contiene varios isozimas (112), y también como el enzima hepático, el cerebral presenta una amplia capacidad para oxidar tanto los aldehídos endógenos como los exógenos, como sería el caso del acetaldehído formado durante la oxidación del etanol.

Sin embargo, como ya se comentó, a pesar de las diferencias existentes en la localización de la actividad de la catalasa y la de ALDH no se invalida la posible acción central del acetaldehído, por ello a continuación se presentan datos genéticos y conductuales que muestran la posible relación que existe entre la actividad de ALDH cerebral y varias de las conductas en las que está implicado el etanol y a la vez su metabolito, el acetaldehído.

\subsubsection{Estudios genéticos.}

Determinar si el acetaldehído desempeña una función en las acciones cerebrales del etanol es una tarea difícil, sin embargo, desde la estrategia genética se ha intentado establecer la posible relación existente entre la actividad de la ALDH y algunos efectos del etanol. Desde esta perspectiva, se ha planteado (113) que las diferencias observadas en la distribución de la actividad en ratas y ratones de cepas diferentes podrían explicar las diferencias obtenidas en algunas conductas como la ingesta voluntaria de alcohol y la incoordinación motora.

Los estudios realizados con diversas cepas (112) indicaron diferencias en la actividad del enzima en neuronas cerebelares de Purkinje, en neuronas corticales, y en el núcleo acumbens, siendo esta actividad mayor en ratas de las cepas long-sleep y en ratas alcohol-tolerant. Similares resultados se encontraron con ratones (58).

Esto podría indicar que la actividad de la ALDH está controlada genéticamente teniendo mediación en la sensibilidad inicial al etanol, en este sentido, la relación existente entre sensibilidad y actividad enzimática podría contribuir a las diferencias existentes en estas cepas de forma que los animales que son más resistentes al etanol tienen un mayor nivel de ALDH en las células cerebelares de Purkinje que los que son más sensibles.

Otras cepas (alcohol-preferring, alcohol-avoiding), se ha obtenido que las segundas presentan no solo una $\mathrm{ADH}$ más eficaz sino también una ALDH menos eficaz $(112,114)$. Así, teniendo en cuenta la analogía existente entre el patrón manifestado en algunos orientales que poseen la ALDH atípica $\left(\mathrm{ALDH}_{2} *_{2}\right)$ y el patrón presentado por las ratas alcohol-avoiding, algunos autores (114) han planteado que la deficiencia en el metabolismo del acetaldehído podría ser el factor subyacente al bajo consumo de etanol desarrollado 
por ratas pertenecientes a esta cepa, al mismo tiempo se plantea que esta cepa podría ser un modelo animal para la deficiencia observada en sujetos humanos en $\mathrm{ALDH}_{2}$. También se ha visto implicación de la actividad de ALDH para oxidar acetaldehído con ratas de la cepa UCh, siendo mayor para las pertenecientes a la cepa UChB, frente a las UChA (96) al parecer debido a que su ALDH mitocondrial de baja Km exhibe una mayor afinidad por el NAD (98).

Estos datos podrían indicar que la alta actividad de la ALDH tiene capacidad para acelerar la eliminación de acetaldehído e incrementar el consumo de etanol reduciendo la respuesta aversiva que se ha observado en algunos animales y humanos.

Por su parte, la genética humana muestra que la deficiencia en ALDH, concretamente en $\mathrm{ALDH}_{2} *_{2}$ (8, para una reciente revisión), supone una mayor concentración de acetaldehído tras la ingesta de etanol frente a sujetos con ALDH normal $\left(\mathrm{ALDH}_{2}\right)$ (115), siendo la acumulación de acetaldehído parcialmente responsable de las bajas dosis de etanol consumidas $(116,117)$. También se ha indicado que la presencia de flushing facial tras el consumo de etanol podría contribuir a un menor consumo (118), sin embargo, los datos obtenidos son contradictorios $(119,120)$.

\subsubsection{Estudios conductuales.}

En general, el pretratamiento con inhibidores de la ALDH produce en sujetos humanos y en animales de laboratorio reducciones en la ingesta de etanol o incluso una supresión en el consumo voluntario de esta sustancia $(121,125,126,127,128,129)$. Así, en animales pretratados con cianamida o algunos de los metabolitos del disulfiram, el aumento en la concentración de acetaldehído se acompaña de una disminución en el consumo de etanol $(129,130)$ o en la actividad locomotora $(86,87,131)$. Algunos autores justifican estos resultados planteando que los altos niveles de acetaldehído en sangre, como consecuencia de la inhibición de ALDH (132) son tóxicos y producen efectos aversivos (133). Sin embargo, tras el uso de estos inhibidores también se ha visto que concentraciones de etanol que no tenían ningún efecto en los sujetos, pasan a ser reforzantes cuando se dan tras la administración de un inhibidor de ALDH (120, 121, 122, 123, 124, 134). Este hecho pone de relieve la implicación de este enzima en los efectos reforzantes del etanol. Los datos que se presentan a continuación van en ese sentido, planteando que la ALDH interacciona con el etanol y metaboliza acetaldehído a nivel central.

Así, aunque tradicionalmente se ha planteado que estos inhibidores reducían la ingesta de alcohol por la acumulación tóxica de acetaldehído, varios estudios han indicado que cuando prevenimos la elevación en sangre de estos niveles mediante el tratamiento concurrente de cianamida y 4- metilpirazol (4-MP) obtene- mos también una supresión de conductas como la ingesta de etanol (129), el CAS (131) o la actividad motora inducidos por etanol $(86,135)$. En este sentido, la supresión ejercida por cianamida, sobre las conductas inducidas por etanol, podría ser debida a la inhibición directa que ejerce sobre la ALDH cerebral y no a la elevación de los niveles de acetaldehído en sangre ocasionados por dicha inhibición ya que el 4-MP, mediante el efecto inhibitorio que ejerce sobre la $\mathrm{ADH}$ disminuye la acumulación periférica de acetaldehído anulando de ese modo la toxicidad periférica. Existen también diversos resultados que indican una relación directa entre actividad de ALDH cerebral y consumo voluntario de etanol en ratas (111, 129, 136, 137, 138).

Por otra parte, aunque no se han encontrado diferencias en los niveles de actividad de ALDH para diferentes cepas de ratas (Long-Evans, Wistar y Sprague-Dawley) los niveles de consumo de alcohol correlacionaron mejor con la actividad de ALDH cerebral para metabolizar aldehído que con la actividad de ALDH hepática (136, 137, 138, 140) pudiendo ser características genéticas previas las que determinarían el consumo posterior de etanol, observando además una correlación alta y positiva entre la capacidad central de oxidar aldehído y la propensión del organismo a autoadministrarse etanol (111). Así, el conjunto de estos datos apoyan la idea de que la actividad de la ALDH cerebral estaría implicada en la regulación del consumo voluntario de etanol. Además, existen datos que indican una función para los enzimas que metabolizan etanol a nivel central en la regulación de la ingesta de alcohol también mediante una regulación, en este caso de los niveles de acetaldehído en el cerebro $(129,141)$

Estos datos nos llevan a plantear que la alteración en la ALDH cerebral por cianamida podría haber potenciado las propiedades farmacológicas de una dosis subumbral de etanol. Como se ha visto, esta idea es congruente con datos anteriores obtenidos con sujetos humanos pretratados con cianamida o disulfiram y tratados posteriormente con bajas dosis de etanol que informan de aumentos en el humor $y$ en la euforia (134). Sin embargo, este resultado no se obtiene para dosis más elevadas (1.2 g/ $\mathrm{kg}$ de etanol), lo que implicaría que la cianamida presenta un efecto dosis-dependiente y de naturaleza bifásica. Como parte de este mecanismo bifásico se ha planteado el concurso de la catalasa en la conversión de cianamida en un metabolito activo $(142,143)$, de forma que las alteraciones en las propiedades discriminables del etanol sean consecuencia de los efectos interactivos producidos por la inhibición en la actividad enzimática de la catalasa y de la ALDH cerebrales.

Desde estudios correlacionales también apoya la implicación de ALDH central en conductas inducidas por etanol. Así, en aquellos animales que presentan una ingesta irregular, es decir, en los que existe una 
mayor variabilidad en el patrón de consumo, la ALDH cerebral predice con mayor fiabilidad el consumo posterior de etanol frente a la ALDH hepática. Este hecho relacionaría a la ALDH cerebral más directamente con la mediación de la preferencia por etanol frente a la ALDH hepática. Así, las diferencias correlacionales, entre ALDH cerebral y hepática en los animales que beben o no con regularidad, sugerirían la posibilidad de que la ALDH cerebral y hepática estén implicadas en diferentes funciones relacionadas con el etanol, regulando aspectos diferentes al destino metabólico del acetaldehído.

Además, también se ha mostrado que la actividad global de ALDH en el cerebro correlacionó con los niveles de ingesta de etanol en ratas bajo una amplia variedad de manipulaciones y condiciones. Obteniendo correlaciones elevadas entre los niveles de consumo voluntario de etanol y la actividad de la ALDH mitocondrial (144). Estos van en la misma dirección que los obtenidos por otros autores (111) y parecen sugerir, como en el caso de la catalasa cerebral, una relación causal no directa entre la actividad enzimática cerebral y la ingestión de etanol (137, 138, 139, 140).

La idea de que la ALDH puede ejercer su acción mediante la regulación de los niveles cerebrales de acetaldehído ha encontrado apoyo también en los resultados de la manipulación de catalasa o ALDH cerebrales, manipulaciones que generan una alteración de las conductas relacionadas con el alcohol (CAS, actividad locomotora, ...).

\section{CONCLUSIONES}

A lo largo de esta revisión se han presentado datos que indican la posibilidad de un metabolismo central del etanol y que, al mismo tiempo, señalan la implicación del acetaldehído en la mediación de algunos de los efectos del etanol, mostrando que el acetaldehído se forma en el cerebro a través de la acción de la catalasa en presencia de etanol y de $\mathrm{H}_{2} \mathrm{O}_{2}$. También, varios estudios han indicado la importancia biológica y conductual del acetaldehído. Además, se ha visto que la actividad central peroxidativa de la catalasa está positivamente correlacionada con la mayor parte de las acciones conductuales conocidas del etanol plasmándose esta relación tanto en estudios con animales como con humanos. Por otra parte, los datos obtenidos a partir del uso de inhibidores de la actividad de la catalasa han dado lugar a una atenuación o bloqueo del consumo voluntario de etanol, así como de otras conductas inducidas por etanol.

En función de todo esto, la explicación más parsimoniosa es la existencia de un mecanismo putativo neurobiológico y conductual por el cual la catalasa podría ejercer su efecto sobre las acciones conductua- les del etanol vía el proceso dependiente del $\mathrm{H}_{2} \mathrm{O}_{2}$ que produce cantidades suficientes de acetaldehído capaces de afectar la concentración de transmisores monoaminos centrales implicados en los procesos motivacionales primarios.

Así, los sistemas enzimáticos formados por la catalasa y la ALDH cerebrales podrían ejercer su acción regulando la formación y la degradación de los niveles de acetaldehído centrales. Esta idea ha sido apoyada por los resultados obtenidos tras la manipulación de estos enzimas que da como resultado una serie de alteraciones en las conductas relacionadas con el etanol. Estos datos sugieren que los enzimas responsables de la formación y degradación del acetaldehído central, quizá mediante la regulación de los niveles de acetaldehído en el cerebro, pueden desempeñar un papel en la mediación de algunas de las acciones farmacológicas del etanol.

En este sentido, si los niveles de acetaldehído en el cerebro son el parámetro fisiológico que determina los efectos del etanol, variaciones en la capacidad metabólica podrían generar un aumento que permitiera diferenciar las respuestas conductuales. Así, la manipulación enzimática del metabolismo del etanol mediante la alteración de algunos de los enzimas implicados -ADH, CYP-450, catalasa y ALDH- podría permitir afirmar no solo la existencia de un metabolismo central del etanol sino también, y más importante si cabe, determinar si es el acetaldehído el responsable de los efectos psicofarmacológicos del etanol.

Por todo ello es necesaria la realización de estudios que permitan consolidar la función de los enzimas que controlan la formación y la degradación de acetaldehído al mismo tiempo que posibilite determinar la implicación indirecta de estos enzimas en los efectos del etanol y la posible participación directa del acetaldehído en algunas de las acciones psicofarmacológicas del etanol.

\section{REFERENCIAS BIBLIOGRÁFICAS}

(1) Tabakoff B, Anderson RA, Ritzmann RF. Brain acetaldehyde after ethanol administration. Biochem Pharmacol. 1976; 25: 1305-09.

(2) Eriksson CJP. Problems and pitfalls in acetaldehyde determinations. Alcohol Clin Exp Res 1980; 4: 22-9.

(3) Deitrich RA. The especifity of ethanol. En: Advances on biomedical alcohol research. 1987; 131-38. Lindros KO. (ed). New York. Pergamon Press.

(4) Sippel HW. The acetaldehyde content in rat brain during ethanol metabolism. J Neurochem 1974; 23: 451-2.

(5) Zimatkin SM. Histochemical study of aldehyde dehydrogenase in the rat CNS. J Neurochem. 1991; 56: 1-11.

(6) Smith BR, Aragon CMG, Amit, Z. Catalase and the production of brain acetaldehyde: a possible mediator of the psychopharmacological effects of ethanol. Addict Biol 1997; 2: 277-89. 
(7) Zimatkin SM, Deitrich RA. Ethanol metabolism in the brain. Addict Biol. 1997; 2: 387-99.

(8) Escarabajal MD. Mecanismos implicados en las conductas inducidas por el alcohol: el papel de los enzimas cerebrales responsables del metabolismo del acetaldehído. 2000; Jaén: Servicio de Publicaciones.

(9) Raskin NH, Sokoloff L. Brain alcohol dehydrogenase. Science. 1968; 162: 131-2.

(10) Raskin NH, Sokoloff L. Alcohol dehydrogenase activityin the rat brain and liver. J Neurochem. 1970; 17: 1677-87.

(11) Raskin NH, Sokoloff L. Enzymes catalyzing ethanol metabolism in neural and somatic tissue of the rat. $J$ Neurochem. 1972; 19: 273-82.

(12) Bühler R, Pestalozzi D, Hess M, Von Wartburg JP. Immunohistochemical localization of alcohol dehydrogenase in human kidney, endocrine organs and brain. Pharmacol Biochem Be. 1983; 18: 1. 55-9.

(13) Kerr, JT, Maxwell DS, Crabb DW. Immunocytochemistry of alcohol dehydrogenase in the rat central nervous system. Alcohol Clin ExpRes. 1989; 13: 730-6.

(14) Tabakoff B, Von Wartburg JP. Separation of aldehyde reductases and alcohol dehydrogenase from brain by affinity chromatography: metabolism of succinic semialdehyde and ethanol. Biochem Biophys Res Comm. 1975; 63: 4. 957-66.

(15) Vallee BL, Bazzone TJ. Isozymes of human liver alcohol dehydrogenase. En: Isozymes, current topics in biological and medical research. 1983; Rattazzi MC, Scandalio JG, Whitt GS. (eds). New York. A. R. Liss. Vol. 8.

(16) Lands WEM. A review of alcohol clearance in humans. Alcohol 1998; 15: 147-60.

(17) Duncan RJS, Kline JE, Sokoloff L. Identity of brain alcohol dehydrogenase. Biochem J. 1976; 153: 561-6.

(18) Iborra FJ, Renau-Piqueras J, Portoles M, Boleda MD, Guerri C, Pares X. Immunocytochemical and biochemical demonstration of formaldehyde dehydrogenase (Class III alcohol dehydrogenase) in the nucleus. J Histochem Cytochem. 1992; 40: 1865-78.

(19) Rout U. Alcohol dehydrogenases in the brain of mice. Alcohol Clin Exp Res. 1992; 16: 2. 286-9.

(20) Giri PR, Linnoila M, O'Neill JB, Goldman D. Distribution and possible metabolic role of class III alcohol dehydrogenase in the human brain. Brain Res. 1989; 481: 131-41.

(21) LieberCS. Ethanol metabolism, cirrhosis and alcoholism. Clin Chim Acta 1997; 257: 59-84

(22) Chernikevich IP, Lomeko IE, Voskoboyev Al, Ostrovsky YM. Evidence on the presence of alcohol dehydrogenase in rat and bovine brain. Neurokhim. 1984; 3: 130-8.

(23) Duester G. A hypothetical mechanism for fetal alcohol syndrome involving ethanol inhibition of retinoic acid synthesis at the alcohol dehydrogenase step. Alcohol Clin ExpRes. 1991; 15: 568-72.

(24) Shean ML, Duester G. The role of alcohol dehydrogenase in retinoic acid homeostasis and fetal alcohol syndrome. Alcohol Alcohol. Suppl. 1983; 2. 51-6.

(25) Koivusalo M, Baumann M, Uotila L. Evidence for the identity of glutathione-dependent formaldehyde dehydrogenase and Class III alcohol dehydrogenase. FEBS Lett. 1989; 257: 105-9.

(26) Hoog JO, Estonius M, Danielsson O. Site-directed mutagenesis and enzyme properies of mammalian alcohol dehydrogenases correlated with their tissue distribution. EXS. 1994; 71: 301-9.

(27) Sasame HA, Ames MM, Nelson SD. Cytochrome P450 and NADPH cytochrome $\mathrm{c}$ reductase in rat brain: formation of reactive catechol metabolites. Biochem Biophys Res Comm. 1977; 78: 919-26.

(28) Ravindranath V, Anandatheerthavarada HK. High activity of cytochrome P450 linked aminopyrine N-demethylase in mouse brain microsomes and associated sex-related differences. Biochem J. 1989; 261: 769-73.

(29) Naslund BMA, Glauman H, Warner M, Gustafsson JA, Hansson T. Cytochrome P450 b and c in the rat brain and pituitary gland. MolPharmacol. 1988; 33: 31-7.

(30) Ravindranath V, Anandatheerthavarada HK, Shankar SK. Xenobiotic metabolism in human brain. Presence of cytochrome P450 and associated mono-oxygenases. Brain Res. 1989; 496: 331-5.

(31) Ghersi-Egea JF, Perrin R, Leininger- Muller B. Subcellular localization of cytochrome P450 and activities of several enzymes responsible for drug metabolism in the human brain. BiochemPharmacol. 1993; 45: 647-58.

(32) Warner M, Gustafsson JA. Effect of ethanol on cytochrome P450 in the rat brain. Proc Nat Ac Sci USA. 1994; 91: 1019-23.

(33) Kholer C, Eriksson LG, Hansson T, Warner M, Gustafsson JA. Immunohistochemical localization of cytochrome P450 in rat brain. NeurosciLett. 1988; 84: 109-14.

(34) Warner M, Kholer C, Hansson T, Gustafsson JA. Regional distribution of cytochrome P450 in the rat brain: spectral quantitation and contribution of $\mathrm{P} 450 \mathrm{~b}$ and c. J Neurochem. 1988; 50: 1057-65.

(35) Morgan ET, Koop DR, Coon MJ. Catalytic activity of cytochrome P450 isozyme $3 a$ isolated from liver microsomes of ethanol-treated rabbits. J BiolChem. 1982; 257: 13951-7.

(36) Hansson T, Tindberg N, Ingelman-Sundberg M, Kohler C. Regional distribution of ethanol inducible cytochrome P450IIE1 in the rat central nervous system. Neuroscience. 1990; 34: 451-63.

(37) Anandatheerthavarada HK, Shankar SK, Bhamre S, Boyd MR, Song BJ, Ravindranath V. Induction of brain cytochrome P450 IIE1 by chronic ethanol treatment. Brain Res. 1993; 601: 279-285.

(38) Sohda T, Shimizu M, Kamimura S, Okumura M. Immunohistochemical demonstration of ethanolinducible P450 2E1 in rat brain. Alcohol Alcohol. Suppl. 1993; 28: 1B. 69-75.

(39) Montoliu C, Valles S, Renau Piqueras J, Guerri C. Ethanol-induced oxygen radical formation and lipid peroxidation in rat brain: effect of chronic alcohol consumption. J Neurochem. 1994; 63: 1855-62.

(40) Bhagwat SV, Boyd MR, Ravindranath V. Brain mitocondrial cytochromes P450: xenobiotic metabolism, presence of 
multiple forms and their selective inducibility. ArchBiochem Biophys. 1995; 320: 73-83.

(41) Tindberg N, Ingelman-Sundberg M. Expression, catalytic activity, and inducibility of cytochrome P450 2E1 (CYP2E1) in the rat central nervous system. J Neurochem. 1996; 67: 2066-73.

(42) Upadhya S, Tirumalai S, Boyd MR, Mori T, Ravindranath V. Cytochrome P4502E (CYP2E1) in brain: Constitutive expression, induction by ethanol and localization by fluorescence in situ hybridation. Arch Biochem Biophys 2000; 373: 23-4.

(43) Hunt WA. Role of acetaldehyde in the actions of ethanol on the brain - A review. Alcohol. 1996; 13: 2. 147-51.

(44) Yasmineh WG, Theologides A. Catalase as a roving scavenger of hydrogen peroxide: A hypothesis. J Lab ClinMed. 1993; 122: 110-4.

(45) McKenna O, Arnold G, Holtzman E. Microperoxisome distribution in the central nervous system of the rat. Brain Res. 1976; 117: 181-94.

(46) Gaunt GL, DeDuve C. Subcellular distribution of Damino acid oxidase and catalase in rat brain. $J$ Neurochem. 1976; 26: 749-59.

(47) Brannan TS, Maker HS, Raes IP. Regional distribution of catalase in the adult rat brain. J Neurochem. 1981; 16:1. 307-9.

(48) Zimatkin SM, Lindros KO. Distribution of catalase in rat brain: aminergic neurons as possible targets for ethanol effects. Alcohol Alcohol. 1996; 31:2. 167-74.

(49) Arnold G, Holtzman E. Microperoxisomes in the central nervous system of the postnatal rat. Brain Res. 1978; 155: 1-17.

(50) Aspberg A, Tottmar O. Development of antioxidant enzymes in rat brain and in reaggregation culture fetal rat brain cells. Dev Brain Res. 1992; 66: 55-8.

(51) Aspberg A, Tottmar O. Ethanol-induced increase in catalase activity in reaggregation cultures of rat brain cells is due to increased oligodendrocyte differentiation. Alcohol Clin ExpRes. 1994; 18: 3. 620-4.

(52) Aspberg A, Soderback M, Tottmar O. Increase in catalase activity in developing rat brain cell reaggregation culturein the presence of ethanol. BiochemPharmacol. 1993; 46: 1873-6.

(53) Hamby-Mason R, Chen JJ, Schenker S, Perez A, Henderson GI. Catalase mediates acetaldehyde formation from ethanol in fetal and neonatal rat brain. Alcohol Clin Exp Res. 1997; 21: 6. 1063-72.

(54) Novikoff AB, Novikoff PM. Microperoxisomes. J Histochem Cytochem. 1973; 21: 963-6.

(55) Zimatkin SM, Lindros KO. Comparison of catalse and aldehyde dehydrogenase distribution in rat brain are aminergic neurons affected by acetaldehyde? Alcohol Clin Exp Res. 1994; 19: 35.

(56) Aragon CMG, Rogan F, Amit Z. Ethanol metabolism in rat brain homogenates by a catalase-H2O2 system. Biochem Pharmacol. 1992a; 44: 93-8.

(57) Gill K, Menez JF, Lucas D, Deitrich RA. Enzymatic production of acetaldehyde from ethanol in rat brain tissue. AlcoholClin Exp Res. 1992; 16: 5. 910-5.
(58) Zimatkin SM, Deitrich RA. aldehyde dehydrogenase activities in the brain of rats and mice genetically selected for different sensitivity to alcohol. Alcohol Clin Exp Res. 1995; 19:5. 1300-6.

(59) Motavkin PA, Okhotin VE, Konovko OO, Zimatkin SM. Localization of aldehyde- and alcohol dehydrogenase in the human spinal cord and brain. Neurosci Behav Physiol. 1990; 2: 79-84.

(60) Amit Z, Stern MH. A further investigation of alcohol preference in the laboratory rat induced by hypothalamic stimulation. Psychopharmacology. 1971; 21: 317-27.

(61) Hashimoto T, Ueha T, Kuriyama T, Katsura M, Kuriyama K. Acetaldehyde-induced alterations in metabolism of monoamines in mouse brain. Alcohol Alcohol. 1989; 24: 91-9.

(62) Heap L, Ward RJ, Abiaka C, Dexter D, Lawlor M, Pratt O, Thomson A, Shaw K, Peters TJ. The influence of brain acetaldehyde on oxidative status, dopamine metabolism, and visual discrimination. Biochem Pharmacol. 1995; 263-70.

(63) Eckardt MJ, File SE, Gessa GL, Grant KA, Guerri C, Hoffman PL, Kalant H, Koob G,F.; Li T-K, Tabakoff B. Effects of moderate alcohol consumption on the central nervous system. Alcohol Clin Exp Res. 1998; 22: 5. 998-1040.

(64) DeMaster EG, Shirota FN, Nagasawa HT. Oxidation of cyanamide by a cumene hydroperoxide-supported catalase reaction yiels cyanide and an inhibitor of aldehyde dehydrogenase. BiochemArc. 1988; 4: 203-7.

(65) Aragon CMG, Stotland LM, Amit Z. Studies on ethanolbrain catalase interaction: Evidence for central ethanol oxidation. Alcohol Clin Exp Res. 1991a; 15: . 165-9.

(66) Cohen G, Sinet PM, Heikkila RE. Ethanol oxidation by rat brain in vivo. Alcohol Clin ExpRes. 1980; 4: 4. 366-70.

(67) Tampier L, Mardones J. Catalase mediated oxidation of ethanol by rat brain homogenates. IRCS. 1979; 7: 389.

(68) Reddy BV, Boyadjieva N, Sarkar DK. Effect of ethanol, propanol, butanol, and catalase enzyme blockers on bendorphin secretion from primary cultures of hypothalamic neurons: evidence for a mediatory role of acetaldehyde in ethanol stimulation of b-endorphin release. Alcohol Clin Exp Res. 1995; 19: 2. 339-44.

(69) Zimatkin SM, Liopo AV, Deitrich, RA. Distribution and kinetics of ethanol metabolism in rat brain. Alcohol Clin Exp Res. 1998; 22: 8. 1623-7.

(70) Lallemand F, Kest W, Ward RJ, De Witte P. Ethanol metabolism in acatalasemic rats. Alcohol Alcohol. 1999; 34: 3. 465.

(71) Gill K, France G, Amit Z. Voluntary ethanol consumption in rats: an examination of blood/brain ethanol levels and behavior. Alcohol Clin Exp Res. 1986; 10: 457-62.

(72) Cross A, Jones OTG. Enzymic mechanisms of superoxide production. Biochim Biophys Acta. 1991; 1057: 281-98.

(73) Pratt AG, Turrens JF. Ascorbate- and hemoglobindependent brain chemiluminescence. Free Radical Bio Med. 1990; 8: 319-25. 
(74) Aragon CMG, Sternklar G, Amit Z. A correlation between voluntary ethanol consumption and brain catalase activity in the rat. Alcohol. 1985a; 2: 353-6.

(75) Amit Z, Aragon CMG. Catalase activity measured in rats naive to ethanol correlates with later voluntary ethanol consumption: possible evidence for a biological marker system of ethanol intake. Psychopharmacology. 1988; 95: 512-5.

(76) Koechling UM, Amit Z. Effects of 3-amino-1,2,4-triazole on brain catalase in the mediation of ethanol consumption in mice. Alcohol. 1994; 11: 3. 235-9.

(77) Aragon CMG, Amit Z. Genetic variation in ethanol sensitivity in C57BL/6 and DBA/2 mice: a further investigation of the differences in brain catalase activity. Ann NY Acad Sci. 1987; 492: 398-400.

(78) Aragon CMG, Amit Z. Differences in ethanol-induced behaviors in normal and acatalasemic mice: systematic examination using a biobehavioral approach. PharmacolBiochem Be. 1993; 44: 547-54.

(79) Aragon CMG, Amit Z. The effects of 3-amino-1,2,4triazole on voluntary ethanol consumption: evidence for brain catalase involvement in the mechanism of action. Neuropharmacology. 1992b; 31: 7. 709-12.

(80) Tampier L, Quintanilla ME, Mardones J. Influence of 3amino-1,2,4-triazole pretreatment on ethanol induced narcosis in rats. IRCS Med Sci. 1979; 7: 390.

(81) Tampier L, Quintanilla ME, Letelier C, Mardones J. Effect of 3-amino-1,2,4-triazole on narcosis time and lethality of ethanol in UChA rats. Alcohol. 1988; 5: 5-8

(82) Aragon CMG, Spivak K, Amit Z. Effects of 3-amino1,2,4-triazole on ethanol-induced narcosis, lethality and hypothermia in rats. Pharmacol Biochem Be. 1991b ; 39: 55-9.

(83) Aragon CMG, Spivak K, Amit Z. Effects of 3-amino1,2,4-triazole on ethanol induced open-field activity: evidence for brain catalase mediation of ethanol's effects. Alcohol Clin Exp Res. 1989; 13:1. 104-8.

(84) Aragon CMG, Spivak K, Amit Z. Blockade of ethanolinduced conditioned taste aversion by 3-amino-1,2,4triazole: evidence for catalase mediated systhesis of acetaldehyde in rat brain. Life Sci. 1985b; 37: 22. 207784.

(85) Escarabajal MD, Miquel M, Aragon CMG. A psychopharmacological study of the relationship between brain catalase activity and ethanol-induced locomotion on mice. J St. Alcohol. 2000; 61: 4. 493-8.

(86) Escarabajal MD, Aragon CMG. The effect of cyanamide and 4-methylpyrazole on the ethanol-induced locomotor activity. Pharmacol Biochem Be. 2002a; 72: 1-2. 389-95.

(87) Escarabajal MD, Aragon CMG. Concurrent administration of diethyldithiocarbamate and 4-methylpyrazole enhances ethanol-induced locomotor activity: the role of brain ALDH. Psychopharmacology. 2002b; 160: 229-43.

(88) Koechling UM, Amit Z. Relationship between blood catalase activity and drinking history in a human population, a possible biological marker of the affinity to consume alcohol. Alcohol Alcohol. 1988; 27:2. 181-8.
(89) Koechling UM, Amit Z, Negrete JC. Family history of alcoholism and the mediation of alcohol intake by catalase. Further evidence for catalase as a marker of the propensity to ingest alcohol. Alcohol Clin Exp Res. 1995; 19: 5. 1096-1104.

(90) Eriksson CJP. The role of acetaldehyde in the actions os alcohol (update 2000). Alcohol Clin Exp Res. 2001; 25: 5. 15S-32S.

(91) Westcott JY, Weiner H, Schultz J, Myers RD. In vivo acetaldehyde in the brain of the rat treated with ethanol. Biochem Pharmacol. 1980; 29: 411-7.

(92) Eysseric H, Gonthier B, Soubeyran A, Bessard G, Saxod $\mathrm{R}$, Barret L. Characterization of the production of acetaldehyde by astrocytes in culture after ethanol exposure. Alcohol Clin Exp Res. 1997; 21: 6. 1018-23.

(93) Clarke DW, Steenaart NAE, Slack CJ, Brien JF. Pharmacokinetics of ethanol and its metabolite, acetaldehyde, and fetotolethality in the third-trimester pregnant guinea pig for oral administration of acute, multiple-dose ethanol. Can J Pharmacol. 1986; 64: 1060-7.

(94) Reddy BV, Sarkar DK. Effect of alcohol, acetaldehyde, and salsonisol on beta-endorphin secretion from the hypothalamic neurons in primary cultures. Alcohol Clin Exp Res. 1993; 17: 6. 1261-7.

(95) Eriksson CJP, Sippel HW. The distribution and metabolism of acetaldehyde in rats during ethanol oxidation-I. The distribution acetaldehyde in liver, brain, blood and breath. Biochem Pharmacol. 1977; 26: 241-7.

(96) Tampier L, Quintanilla ME, Contreras S, SegoviaRequelme N, Mardones J. Biological similarities and differences between rats genetically different in alcohol preference. Alcohol Alcohol. 1984; 19: 3. 203-9.

(97) Tampier L, Quintanilla ME, Mardones J. Methanol, ethanol and acetaldehyde oxidation rates by homogenates of different brain regions of UChA and UChB rats. IRCS Med Sci. 1980; 8: 157-8.

(98) Quintanilla ME, Tampier L. Acetaldehyde metabolism by brain mitochondria from UChA and UChB rats. Alcohol. 1995; 12: 6. 519-24.

(99) Eriksson CJP. Ethanol and acetaldehyde metabolism in rat strains genetically selected for their ethanol preference. Biochem Pharmacol. 1973; 22: 2283-92.

(100) Zeiner AR, Kegg PS, Blackburn M, Stratton R. Gender differences in peak acetaldehyde concentration after an acute dose of ethanol. Neurobehav Toxicol Teratol. 1983; 5: 2. 201-4.

(101) Lindros KO. Research on experimental and inborn alterations of acetaldehyde metabolism. Implications for treatment of alcoholism. The 2nd Malms Symposium on Alcohol. 1984; 115-125.

(102) Brown Z, Amit Z, Rockman GE. Intraventricular selfadministration of acetaldehyde but not ethanol in naive laboratory rats. Psychopharmacology. 1979; 64: 271-6.

(103) Brown ZW, Amit Z, Smith BR. Intraventricular selfadministration of acetaldehyde and voluntary consumption of ethanol in rats. Behav Neural Biol. 1980; 28: 150-5. 
(104) Rodd-Henricks ZA, Zaffaroni A, Goldstein A, McBride WJ, LiTK. Alcohol Preferring $(P)$ rats self-administer acetaldehyde directely into de posterior VTA (abstract). Alcohol Clin Exp Res Suppl 2000; 5: 24: 52A.

(105) Smith BR, Amit Z, Splawinsky J. Conditioned place preference induced by intraventricular infusions of acetaldehyde. Alcohol. 1984; 1: 193-5.

(106) Quertemont E, De Witte P. Conditioned stimulus preference after acetaldehyde but not ethanol injections. Pharmacol Biochem Be 2001; 68: 449-54.

(107) Inoue K, Lindros KO. Subcellular distribution of human brain aldehyde dehydrogenase. J Neurochem. 1982; 38: 4. 884-8.

(108) Koivula T, Turner AJ, Huttunen M, Koivusalo M. Subcellular and perisynaptic distribution of rat brain aldehyde dehydrogenase activity. J Neurochem. 1981; 36: 1893-7.

(109) Zimatkin SM, Rout UK, Koivusalo M, BŸhler R, Lindros $\mathrm{KO}$. Regional distribution of low-Km mitochondrial aldehyde dehydrogenase in the rat central nervous system. Alcohol Clin Exp Res. 1992; 16: 1162-7.

(110) Weiner H, Ardelt B. Distribution and properties of acetaldehyde dehydrogenase in regions of rat brain. $J$ Neurochem. 1984; 42: 1. 109-15.

(111) Socaransky SM, Aragon CMG, Amit Z. Brain ALDH as a possible modulator of voluntary ethanol intake. Alcohol. 1985; 2: 2. 361-5.

(112) Koivula T, Koivusalo M, Lindros KO. Liver aldehyde and alcohol dehydrogenase activities in rat strains genetically selected for their ethanol preference. Biochem Pharmacol. 1975; 24: 1807-11.

(113) Zimatkin SM, Lindros KO. A histochemical study of the distribution of aldehyde dehydrogenase activity in brain structures of rats with genetically different alcoholrelated behaviour. Alcohol. 1989; 6: 4. 321-5.

(114) Koivisto T, Eriksson CJP. Hepatic aldehyde and alcohol dedydrogenases in alcohol-preferring and alcoholavoiding rat lines. Biochem Pharmacol. 1994; 48: 1551-8.

(115) Mizoi Y, Kogama M, Fukunaga T, Ueno Y, Adachi J, Fujiwara S. Polymorphism of acetaldehyde dehydrogenase and ethanol elimination. Alcohol. 1985; 2: 3. 393-6.

(116) Agarwal DP, Eckey R, Harada S, Goedde HW. Basis of acetaldehyde dehydrogenase deficiency in Orientals immunochemical studies. Alcohol. 1984; 1:2. 111-8.

(117) Tu GC, Israel Y. Alcohol consumption by Orientals in North America is predicted largely by a single gene. Behav Gene. 1995; 25: 1. 59-65.

(118) Wall TL, Thomasson HR, Schukit MA, Ehlers CL. Subjetive feelings of alcohol intoxication in Asians with genetic variations of ALDH2 alleles. Alcohol Clin Exp Res. 1992; 16: 5. 991-5.

(119) Wolff P. Ethnic differences in alcohol sensitivity. Science. 1972; 175: 449-50.

(120) Peachey JE, Zilm D, Robinson GM, Jacob M, Cappell H. A placebo-controlled double-blind comparative clinical study of the disulfiram- and calcium carbimideacetaldehyde mediated ethanol reactions in social drinkers. Alcohol Clin Exp Res. 1983; 7: 2. 180-7.
(121) Amit Z, Brown ZW, Amir S, Smith B, Sutherland EA. Behavioral assessment of the role of acetaldehyde in the medioation of alcohol intake in animal and humans. En: Animal Models in Alcohol Research. 1980; 159-165; Eriksson V, Sinclair JD, Kiianmaa K. (eds). New York. Academic Press.

(122) Peachey JE, Zilm D, Cappell H. Comparative study of the disulfiram-ethanol reaction and the carbimideethanol reaction in nonalcoholic men. II: Effects of repeated drinks. Clin Pharmacol Ther. 1981a; 29: 2. 271.

(123) Peachey JE, Zilm D, Cappell H, Robertson G. Comparative study of the disulfiram-ethanol reaction and the carbimide-ethanol reaction in nonalcoholic men. I: Effects of initial alcohol exposure. 1981b; 29: 2. 271.

(124) Peachey JE, Brien JF, Zilm DH, Loomis CW, Hemy MF, Maglana SM. The calcium cyanamide-ethanol interaction in man. Effects of repeated ethanol administration. J St. Alcohol. 1981c; 42: 3. 208-16.

(125) Koe BK, Tenen SS. Inhibiting action of n-butyraldoxime on ethanol metabolism and on natural preference of C57BL mice. J Pharmacol Exp Ther. 1970; 174: 434-49.

(126) Reed TE, Kalant H, Gibbins RJ, Kapur BM, Rankin JG. Alcohol and acetaldehyde metabolism in Caucasians, Chinese and Amerinds. Can Med Ass. 1976; 115: 851-5.

(127) Mottin JL. Drug-induced attenuation of alcohol consumption. A review and evaluation of claimed, potential or current therapies. J St. Alcohol. 1973; 34: 444-72.

(128) Eriksson CJP, Deitrich RA Evidence against a biphasic effect of acetaldehyde on voluntary ethanol consumption in rats. Pharmacol Biochem Be Suppl. 1980; 13: 1. 291-6.

(129) Sinclair JD, Lindros KO. Supression of alcohol drinking with brain aldehyde dehydrogenase inhibition. Pharmacol Biochem Be. 1981; 14: 3. 377-83.

(130) Sinclair JD, Lindros KO, Tehro K. Aldehyde dehydrogenase inhibitors and voluntary ethanol drinking by rats. En: Alcohol and Aldehyde Metabolizing Systems. 1980; Vol. 4. 481-7; Thurman RG. (ed). New York. Plenum Press.

(131) Spivak K, Aragon CMG, Amit Z. Alterations in brain aldehyde dehydrogenase activity modify the locomotor effects produced by ethanol in rats. Alcohol Drug Res. 1987b; 7: 481-91.

(132) Deitrich RA, Troxell PA, Worth WS, Erwin VG. Inhibition of aldehyde dehydrogenase in brain and liver by cyanamide. Biochem Pharmacol. 1976; 25: 2733-7.

(133) Sinclair JD. Drugs to decrease alcohol drinking. Ann Med. 1990; 22: 5. 357-62.

(134) Brown ZW, Amit Z, Smith BR, Sutherland EA, Selvaggi N. Alcohol-induced euphoria enhanced by disulfiram and calcium carbimide. Alcohol Clin Exp Res. 1983; 7: 276-8.

(135) Spivak K, Aragon CMG, Amit Z. Alterations in brain aldehyde dehydrogenase activity modify ethanol induced conditioned taste aversion. Alcohol Clin Exp Res. 1987a; 11: 513-7. 
(136) Amir S. Brain and liver aldehyde dehydrogenase: relations to ethanol consumption in wistar rats. Neuropharmacology. 1977; 16: 781-4.

(137) Amir S. Brain and liver aldehyde dehydrogenase activity and voluntary ethanol consumption by rats: Relations to strain, sex and age. Psychopharmacology. 1978a; 57: 97-102.

(138) Socaransky SM, Aragon CMG, Amit Z, Blander A. Higher correlation of ethanol consumption with brain than liver ALDH in three strains of rats. Psychopharmacology. 1984; 84: 2. 250-3.

(139) Amir S. Brain aldehyde dehydrogenase: Adaptative increase following prolonged ethanol administration in rats. Neuropharmacology. 1978b; 17: 463-7.

(140) Sinclair JD, Lindros KO. Acetaldehyde accumulation and voluntary ethanol intake by rats. Alcohol Clin Exp Res. 1979; 3: 276-85.
(141) Spivak K, Amit Z. The role of acetaldehyde-metabolizing enzymes in the mediation of ethanol consumption: An investigation using a simulated drinking bout. Alcohol Alcohol Suppl. 1987; 22: 1. 361-5.

(142) Cederbaum Al, Dicker E. Inhibition of the peroxidatic activity of catalase toward alcohols by the aldehyde dehydrogenase inhibitor cyanamide. Toxicol Lett. 1985; 29: 107-14.

(143) DeMaster EG, Redfern B, Shirota FN, Nagasawa HT. Differential inhibition of rat tissue catalase by cyanamide. Biocheml Pharmacol. 1986; 35: 13. 2081-5.

(144) Aragon CMG, Amit Z. A two dimensional model of alcohol consumption: Possible interaction of brain catalase and aldehyde dehydrogenase. Alcohol. 1985c; 2: 357-60. 